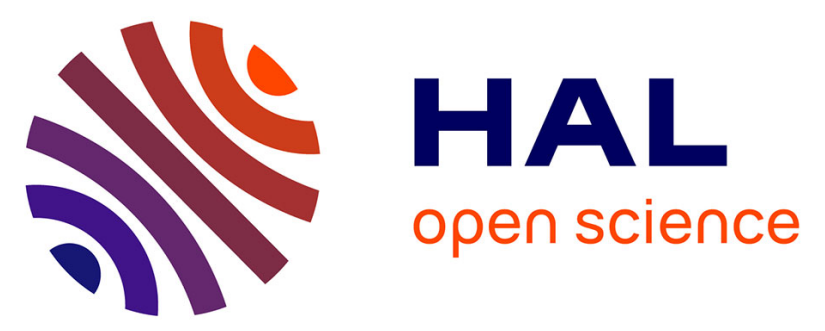

\title{
Sensor-integrated fluorescent microarray for ultrahigh sensitivity direct-imaging bioassays: Role of a high rejection of excitation light
}

Lucio Martinelli, Houtai Choumane, Khoi-Nguyen Ha, Gabriel Sagarzazu, Carole Goutel, Claude Weisbuch, Thierry Gacoin, Henri Benisty

\section{To cite this version:}

Lucio Martinelli, Houtai Choumane, Khoi-Nguyen Ha, Gabriel Sagarzazu, Carole Goutel, et al.. Sensor-integrated fluorescent microarray for ultrahigh sensitivity direct-imaging bioassays: Role of a high rejection of excitation light. Applied Physics Letters, 2007, 91, pp.083901. 10.1063/1.2767209 . hal-00875750

\section{HAL Id: hal-00875750 \\ https://hal-iogs.archives-ouvertes.fr/hal-00875750}

Submitted on 22 Oct 2013

HAL is a multi-disciplinary open access archive for the deposit and dissemination of scientific research documents, whether they are published or not. The documents may come from teaching and research institutions in France or abroad, or from public or private research centers.
L'archive ouverte pluridisciplinaire HAL, est destinée au dépôt et à la diffusion de documents scientifiques de niveau recherche, publiés ou non, émanant des établissements d'enseignement et de recherche français ou étrangers, des laboratoires publics ou privés. 


\title{
Sensor-integrated fluorescent microarray for ultrahigh sensitivity direct-imaging bioassays: Role of a high rejection of excitation light
}

\author{
Lucio Martinelli, Houtai Choumane, Khoi-Nguyen Ha, Gabriel Sagarzazu, \\ Carole Goutel, and Claude Weisbuch \\ Genewave, XTEC, Ecole Polytechnique Campus, 91120 Palaiseau, France \\ Thierry Gacoin \\ Laboratoire de Physique de la Matière Condensée, Ecole Polytechnique, 91128 Palaiseau cedex, France \\ Henri Benisty ${ }^{\text {a) }}$ \\ Laboratoire Charles Fabry, CNRS, Institut d'Optique Graduate School, Campus Polytechnique, \\ 91127 Palaiseau cedex, France
}

(Received 12 April 2007; accepted 9 July 2007; published online 20 August 2007)

\begin{abstract}
Fluorescent microarrays exploit fluorescent labeled targets bound to immobilized biomolecular probes. Their signal-to-noise ratio is limited by the collection aperture in common confocal geometries. Taking advantage of a very high rejection filter deposited onto a silicon arrayed detector (coupled-charge device or complementary metal-oxide semiconductor), it is demonstrated that a highly compact lens-free assay with photon collection of order unity operates with a 30-fold improvement over a conventional (substrate + free-space optics) scheme. Through analysis of improvements over the present demonstrator, a single molecule per pixel sensitivity is predicted. (C) 2007 American Institute of Physics. [DOI: 10.1063/1.2767209]
\end{abstract}

For biomolecular sensors, the quest for ultimate sensitivity, miniaturization, and low cost are major driving forces. Among sensing technologies, fluorescence is the key technique for ultimate sensitivity with its single molecule detection capability. It is widely used in microarrays ${ }^{1}$ probing thousands of "spots" that reveal deoxyribonucleic-acid (DNA)-related information (notably differential genetic expression), making accuracy and high sensitivity key demands. Fluorescence techniques, however, tend to require bulky readers, for example confocal microscopes associated with mechanical scanning.

It has been recognized that direct imaging obtained by spotting directly on an image sensor brings both compactness and high collection efficiency. ${ }^{2-6}$ However, the first demonstrations relied on radioactive arrays ${ }^{2,3}$ or on bioluminescence $e^{4,5}$ where the exciting light rejection is not an issue. More recent realisations involved a sensor and a multilayered dielectric filter on an $a$-Si photodetector ${ }^{6}$ or with a CdS filter on a Si detector. ${ }^{7}$ Other schemes integrated microarray, light source, and specific detector, ${ }^{8,9}$ but they led to large amounts of stray light, and low sensitivity. ${ }^{10}$ In contrast with the previous works based on dedicated image sensors, we show that a detection based on fluorescently labeled species directly deposited on a filter, itself directly deposited on a standard, commercial solid-state imager achieves impressive improvements in sensitivity (30 times) over usual imaging systems along with webcam camera compacity. Experimental threshold detection of quantum-dot labeled probes is found at around $10 \mathrm{dots} / \mu \mathrm{m}^{2}$ areal density not yet at the detection limit. The analysis indicates guidelines for a sensitivity of 1 fluorophore $/ 10 \mu \mathrm{m}^{2}$ pixel, taking into account rejection of excitation light, absorption cross section of the fluorophore, bleaching sensitivity (number of photons emitted per fluorescent label before it is destroyed), and array

a)Electronic mail: henri.benisty@institutoptique.fr readout noise. This study can also be used to estimate the allowed amount of filter autofluorescence.

Our scheme for ultrasensitive detection is sketched in Fig. 1(a). A high-rejection wavelength selective filter lies directly on an arrayed detector, here of the charge-coupled device (CCD) type. Fluorophore-labeled species are spotted directly onto the filter, photoexcited by an impinging beam. Their fluorescence at $\lambda_{\text {emi }}$ is detected through the filter. This is at variance with the free-space optics scheme [Fig. 1(b)], where collection occurs through lenses and the rejection ("emission") filter lies on the optical path.

In the data reported here we use commercial CCD cameras (either Kodak KAF 1602-NE or e2v TH-7899M). Similar results were obtained on low-cost complementary metaloxide semiconductor (CMOS) webcam cameras. The
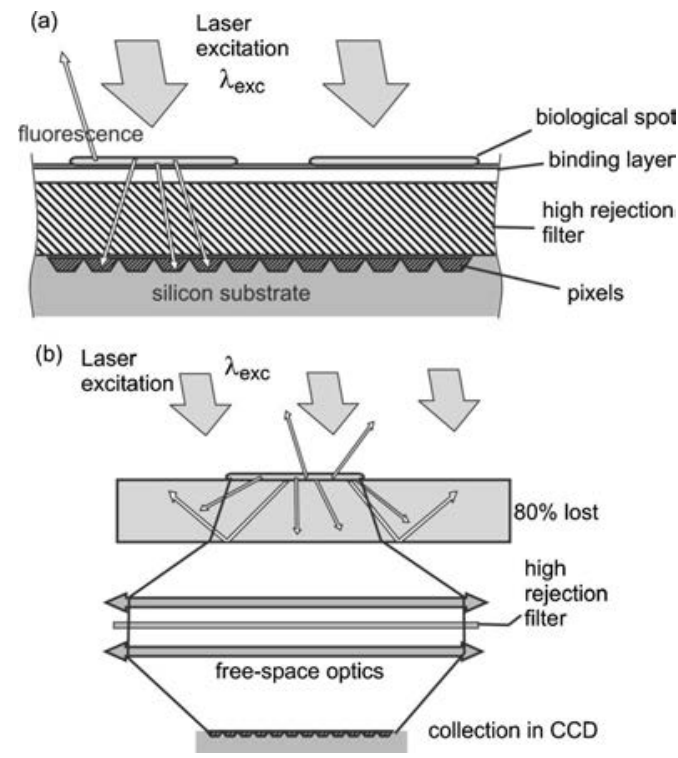

FIG. 1. Schematics of (a) the direct-imaging integrated microarray sensor and (b) of the free-space imaging system it replaces. 


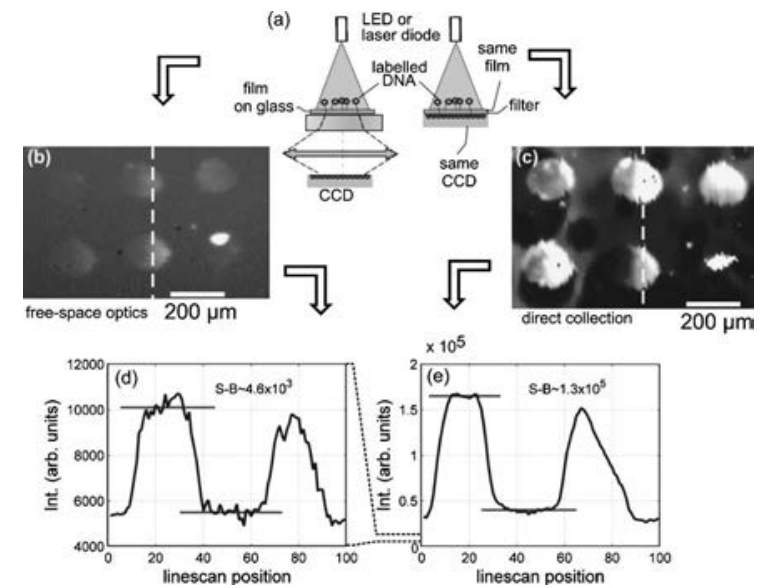

FIG. 2. (a) Schematics of the experiment: the same spots are measured either with a filter on glass (where the filter was elaborated) by free-space optics or imaged with the filter directly onto the same CCD chip; (b) image by free-space optics (dark except at a pinhole); (c) direct detection image; [(d) and (e)] respective line scans of (b) and (c) where indicated by the dashed lines, showing a gain (signal to background) by a factor of $\sim 27$.

selective filter is directly spin coated on the finished camera, and then annealed, or is lifted off from a glass substrate and transferred to the camera. It consists of a polyimide layer doped with a chromium-based dye (typical thicknesses are $6 \mu \mathrm{m}$ and dye concentration is $50 \%$ in volume in the polymer solution), leading to a transmission coefficient lower than $\sim 10^{-6}$ at $\lambda_{\text {exc }}$ and a $60 \%$ transmission at $\lambda_{\text {emi. }}$. Increasing the filter thickness to still improve exciting light rejection is of no help as the residual filter fluorescence is also in the $10^{-6}$ range. We either use fluorescent dyes $\left(\mathrm{Cy} 3^{\mathrm{TM}}\right)$ or quantum dots (QDs) as fluorophores, the latter being preferred here because of their larger absorption cross section and larger Stokes shift. Since the selective filter used has a nonabrupt absorption edge, a larger Stokes shift provides a better compromise between excitation absorption and fluorescence transmission. Thus, using QD emission at $\lambda_{\text {emi }}=800 \mathrm{~nm}$ (QD 800 from QD Corporation) and $\lambda_{\mathrm{exc}}=532 \mathrm{~nm}$ result in a rejection ratio of ratio of $10^{6}$ for QDs (in the following, this includes filter fluorescence), while for $\mathrm{Cy} 3$ excitation at $532 \mathrm{~nm}$ and emission at $\sim 600 \mathrm{~nm}$, the filter yields a rejection of a few $10^{4}$. This favors QDs inasmuch as they do not introduce other drawbacks. ${ }^{11}$

Figure 2 shows a comparison of signals obtained either from Cy3 labeled oligonucleotides spotted filter on glass imaged through a classic optical system [numerical aperture $(\mathrm{NA})=0.5]$ on the Kodak CCD $[(\mathrm{a})$ and (b) $]$ or through a direct imaging after the film and spots have been transferred on the same imager [(a) and (c)]. These images display two remarkable features. (i) The direct imaging shows that there is no crosstalk between spots. This is a basic benefit of the low thickness (6 $\mu \mathrm{m}$ here). It also discards crosstalk arising through guided modes in the filter or other layers atop. (ii) The quantitative analysis of the direct image [Fig. 2(c)] shows that the fluorescence signal is roughly 30 times larger than that obtained in the free-space imaging system. This is attributed to the difference in collection efficiency. While it is typically only $2 \%$ in the imaging system (fraction of light emitted into air, collected fraction, transmission of filter and optics), it reaches $60 \%$ in the direct imaging mode (very high emitted fraction into the layers and silicon due to their higher index, almost unity collected fraction, only diminished by reflection at the dielectric silicon interface and by filter trans- mission). Inhomogeneities in the direct image are due to the variations in absorbing film thickness.

Other experiments were performed on the e2v CCD camera where the film was spun on directly, and where hybridization was carried out after functionalizing the surface with amino silanes in the gas phase and spotting 25-mer oligonucleotides. The CCD was placed in a special hybridization chamber. Hybridization results were very similar to those obtained with standard glass slides.

On the same camera, we evaluated the sensitivity of the sensor. Spots with a diameter of $250 \mu \mathrm{m}$, i.e., a surface $S$ $\sim 6 \times 10^{4} \mu \mathrm{m}^{2}$, were obtained from $1 \mathrm{nl}$ droplets with variable QDs concentrations (QD 800) down to $C=4 \mathrm{nM}$. For the lowest concentration used, the observed signal-to-noise ratio is 4 which implies a limit fluorophore detectable density of 10 fluorophores $/ \mu \mathrm{m}^{2}$. With a Cy3 labeling, only the fluorescence spectral tail traverses our filter and the sensitivity is degraded by a factor of about 30 .

This is not the ultimate sensitivity of this detection scheme. Using a lower fluorescence absorbing dye in the filter, or combinations of filter layers with shifted absorption edges (hence selective absorption of the first layer's fluorescence background), one should be able to diminish fluorescence background by two orders of magnitude. Another way to get more signal and diminish background at a given excitation intensity is to implement on the filter a distributed Bragg reflector (DBR) structure centered at the excitation wavelength with a low-index quarter wavelength layer on top. ${ }^{12}$ In that case, due to the constructive interference, excitation intensity can be improved by a factor of $4-6$, which is positively compounded by the diminished DBR-transmitted excitation light, improving over the present situation by similar two orders of magnitude. The limit sensitivity can then reach the single molecule level (one molecule over a single $10 \mu \mathrm{m}^{2}$ pixel), provided that the dark current of the imager is small enough.

To get the generic impact of parameters on direct imaging fluorescence systems, we carry a quantitative analysis, taking as parameters the rejection ratio (which also includes parasitic fluorescence light) and the fluorophore absorption cross section $\sigma$ and its quantum yield $\eta$.

An excitation photon fluence $\phi_{\text {exc }}\left(\mathrm{J} / \mathrm{m}^{2}\right)$ impinges on an areal density $C$ of fluorophores. If the photon collection efficiency by the collection optics is denoted $\eta_{\text {coll }}$ (taking into account the responsivity of the pixel), the useful collected signal from a single molecule emitting $N_{\text {emiss }}$ photons is

$$
N_{\text {coll }}=N_{\text {emiss }} \eta_{\text {coll }}=\sigma \eta / h \nu \phi_{\text {exc }} \eta_{\text {coll }} \text {. }
$$

For an areal concentration $C$ and a pixel area $S$, the photoelectron number per pixel is just $C S N_{\text {coll }}$. This signal competes with insufficiently rejected excitation photons whose signal is $N_{\text {bck }}=S \phi_{\text {exc }} /(R h \nu)$, where $R(\gg 1)$ is the excitation rejection ratio of the deposited filter: we use $\eta_{\text {coll }}$ $\sim 1$ for these photons as their flux is still directional, and we include the internal efficiency at $\lambda_{\text {exc }}(\sim 0.5)$ in $R$. Common commercial filters feature $R \sim 10^{8}$.

Going to signal-to-noise ratio (SNR), the noise associated with $N_{\text {bck }}$ is a first detection limit. However, for the high sensitivities, down to a few molecules detection, a conservative limit is rather $N_{\text {coll }}=N_{\text {bck }}$.

Then, the limit condition for detection reads $R C \sigma \eta \eta_{\text {coll }}$ $=1$, corresponding to straight lines in Fig. 3 for various rejection ratios $R$ in the $C$ vs $\sigma$ plane (independent of $S$ ). We to AlP license or copyright, see http://apl.aip.org/apl/copyright.jsp 

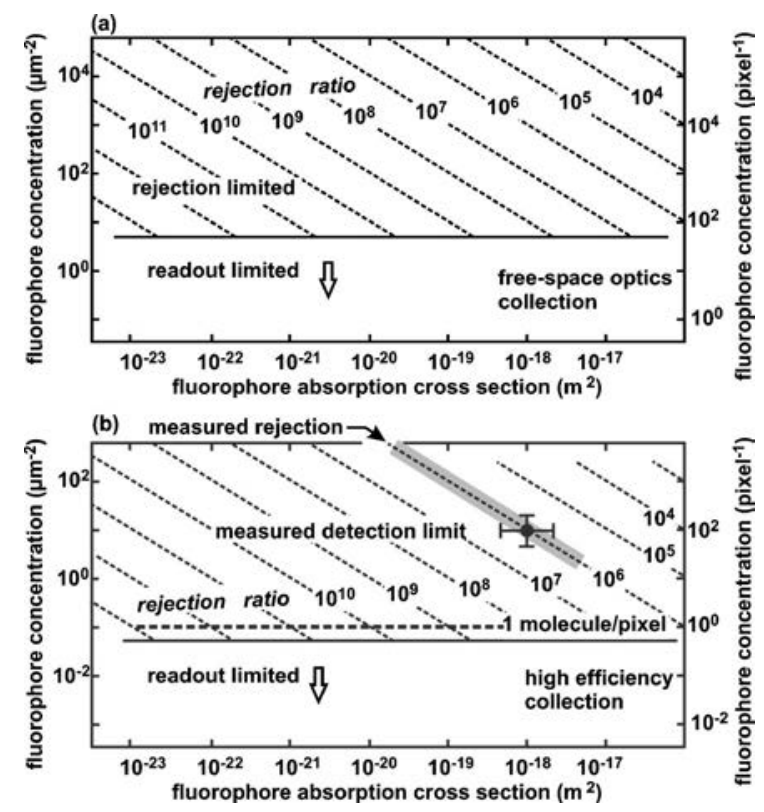

FIG. 3. Performance limit of two configurations with (a) basic free-space optics configuration $(0.3 \%$ collection) and (b) on-CCD integrated configuration (30\% collection), the bolder dotted lines indicate 1 molecule $/ 10 \mu \mathrm{m}^{2}$ pixel for the (b) configuration. The "readout limited" region indicates limit detection for 300 fluorescence photons/molecule before $0.1 \%$ bleaching, a typical practical value. The measured rejection ratio defines the detection limit line (in gray) in terms of fluorophore concentration vs cross section.

use $\eta=0.3$ to emphasize the importance of $\eta_{\text {coll }}$ and show two graphs with (a) $\eta_{\text {coll }}=0.003$, a typical value of a freespace microarray reader (with a NA $\sim 0.25-0.30$, the typical space optics compatible with $\mathrm{cm}^{2}$-scale imaging, comparable to our system), and (b) $\eta_{\text {coll }}=0.3$, the good value of Fig. 1 scheme (within the assumption of good laser blocking and sufficient Stokes shift.). The diagrams suggest that systems with high rejection ratio, large absorption cross sections, and high collection efficiencies can attain single molecule detection. To be more complete we have to consider also the detector readout noise $N_{e}$, a characteristic of readout detectors. We shall use here $N_{e} \sim 50$ (typical of arrays at room temperature). The overall detection limit can be taken as:

$C_{\min } S \frac{\sigma \eta}{h \nu} \phi_{\text {exc }} \eta_{\text {coll }}>($ SNR margin $) \times \max \left(N_{\text {bck }}, N_{e}\right)$,

noting that a more detailed analysis is incorporated in the factor (SNR margin) taken as unity in Fig. 3.

At this point, more excitation fluence can always beat the imager noise. However, there is a limit to the number of photons that a fluorophore can emit, due to bleaching, a common occurrence in the case of organic fluorophores such as dyes. In a practical experiment, one restricts emission conservatively at $0.1 \%$ of the total photons emitted by a typical dye before bleaching. Taking a typical number of emitted photons before bleaching at $3 \times 10^{5}$, we get a value of $N_{\text {emiss }}=\sigma \eta \phi_{\text {exc }} / h \nu=300$ and thus, in Eq. (2) a value of $C S \eta_{\text {coll }}$ of $1 / 6\left(300 / N_{e}\right.$ with $\left.N_{e} \sim 50\right)$ when the number of photoelectrons equals the readout noise. This sets the detection limit at $1 / 2$ fluorophore per pixel, with $\eta_{\text {coll }}=0.3$. The regions of the plane marked as readout limited are beyond a limit never reached in practice, unless the fluorophore has extremely poor bleaching properties and the filter has remarkably high rejection. Clearly, with these conservative es- timates, single molecule detection is within reach. Help of dielectric layers (that achieve laser blocking up to $10^{7}$ in good conditions) or chip cooling to reduce dark current are the options for the highest sensitivity obtainment.

The calibrated experimental point is shown in Fig. 3(b), using QD's cross section $\sigma$ from Ref. 13, pointing to the validity of the analysis. Note that we selected standard values of parameters to carry out this analysis. Variants (existence of imager dark current, absorber fluorescence, etc.) would not significantly alter the results.

In conclusion, we demonstrate that the integrated microarray/filter/imager system has remarkable properties provided that the rejection ratio and background fluorescence of the filtering element are good enough. The sensitivity is similar to that of the best confocal scanning systems (in the tens of fluorophore per $\mu \mathrm{m}^{2}$ range), while attaining remarkable features in terms of compacity and cost thanks to the use of standard imaging chips. In addition, improvements should bring the sensitivity to the single molecule level. The advent of integrated hybridization-readout systems in standard silicon technology helps incorporating more functions: the entire control and communication system can be integrated, with a standard interface (e.g., universal service bus) to a computer. The biochip data, from fabrication to function (built-in biological test, quality controls, and diagnostic software), provide stand-alone full-biochip operation. Other opportunities provided by Si technology are built-in electrodes for the fabrication of probes or high-efficiency hybridization, thanks to electrokinetic control of DNA or protein fragment motion, or other desired lab-on-chip functions.

The authors thank the Genewave team. This work has been partly supported by the EU INDIGO IST project.

${ }^{1}$ M. Schena, Microarray Analysis (Wiley, Hoboken, New Jersey, 2003).

${ }^{2}$ M. D. Eggers, M. E. Hogan, R. K. Reich, J. B. Lamture, K. Beattie, M. A. Hollis, D. J. Ehrlich, B. B. Kosicki, J. M. Shumaker, R. S. Varam, B. E. Burke, A. Murphy, and D. D. Rathman, Proc. SPIE 1891, 113 (1993).

${ }^{3}$ J. B. Lamture, K. Beattie, B. E. Burke, M. D. Eggers, D. J. Ehrlich, R. Fowler, M. A. Hollis, B. B. Kosicki, R. K. Reich, S. R. Smith, R. S. Varma, and M. E. Hogan, Nucleic Acids Res. 22, 2121 (1994).

${ }^{4}$ U. Lu, B. C.-P. Hu, Y.-C. Shih, C.-Y. Wu, and Y.-S. Yang, Biosens. Bioelectron. 19, 1185 (2004).

${ }^{5}$ K. Salama, H. Eltoukhy, A. Hassibi, and A. El Gamal, Biosens. Bioelectron. 19, 1377 (2004).

${ }^{6}$ F. Fixe, V. Chu, D. M. F. Prazeres, and J. P. Conde, Nucleic Acids Res. 32, e70 (2004).

${ }^{7}$ J. A. Chediak, Z. S. Luo, J. G. Seo, N. Cheung, L. P. Lee, and T. D. Sands, Sens. Actuators, A 111, 1 (2004).

${ }^{8}$ V. Savvate'ev, Z. Chen-Esterlit, J. W. Aylott, B. Choudhury, C. H. Kim, L. Zou, J. H. Friedl, R. Shinar, J. Shinar, and R. Kopelman, Appl. Phys. Lett. 81, 4652 (2002).

${ }^{9}$ E. Thrush, O. Levi, W. Ha, G. Carey, L. Cook, J. Deich, S. J. Smith, W. E. Moerner, and J. S. Harris, Jr., IEEE J. Quantum Electron. 40, 491 (2004).

${ }^{10}$ E. Thrush, O. Levi, L. J. Cook, J. Deich, S. J. Smith, W. E. Moerner, and J. S. Harris, Jr., Proc. SPIE 5318, 59 (2004).

${ }^{11}$ The use of QDs in DNA labeling (using either direct or indirect strategies) is increasing on account of their photostability and fluorescence yield, see, e.g., W. R. Algar and U. J. Krull, Langmuir 22, 11346 (2006); R. Bakalova, Z. Zhelev, H. Ohba, and Y. Baba, J. Am. Chem. Soc. 127, 11328 (2005); H. Peng, L. Zhang, T. H. Kjällman, C. Soeller, and J. Travas-Sejdic, ibid. 129, 3048 (2007); N. C. Cady, A. D. Strickland, and C. A. Batt, Mol. Cell. Probes 21, 116 (2007).

${ }^{12}$ H. Choumane, N. Ha, C. Nelep, A. Chardon, G.-O. Reymond, C. Goutel, G. Cerovic, F. Vallet, C. Weisbuch, and H. Benisty, Appl. Phys. Lett. 87, 031102 (2005).

${ }^{13}$ A. Leatherdale, W.-K. Woo, F. V. Mikulec, and M. G. Bawendi, J. Phys. Chem. 106, 7619 (2002). 